Article

\title{
Operation of a MOEMS Deformable Mirror in Cryo: Challenges and Results
}

\author{
Frederic Zamkotsian ${ }^{1, *}$, Patrick Lanzoni ${ }^{1}$, Rudy Barette ${ }^{1}$, Michael Helmbrecht ${ }^{2}$, \\ Franck Marchis $^{3}$ (i) and Alex Teichman ${ }^{2}$ \\ 1 Aix Marseille Univ, CNRS, LAM, Laboratoire d'Astrophysique de Marseille, 38 rue Frederic Joliot Curie, \\ 13388 Marseille CEDEX 13, France; patrick.lanzoni@lam.fr (P.L.); rudy.barette@lam.fr (R.B.) \\ 2 Iris AO, 2930 Shattuck Avenue \#304, Berkeley, CA 94705, USA; info@irisao.com or \\ michael.helmbrecht@irisao.com (M.H.); alex.teichman@irisao.com (A.T.) \\ 3 Carl Sagan Center, SETI Institute, 189 Bernardo Ave, Mountain View, CA 94043, USA; fmarchis@seti.org \\ * Correspondence: frederic.zamkotsian@lam.fr; Tel.: +33-4-9504-4151
}

Received: 24 May 2017; Accepted: 14 July 2017; Published: 27 July 2017

\begin{abstract}
Micro-opto-electro-mechanical systems (MOEMS) Deformable Mirrors (DM) are key components for next generation optical instruments implementing innovative adaptive optics systems, both in existing telescopes and in the future ELTs. Characterizing these components well is critical for next generation instruments. This is done by interferometry, including surface quality measurement in static and dynamical modes, at ambient and in vacuum/cryo. We use a compact cryo-vacuum chamber designed for reaching 10-6 mbar and $160 \mathrm{~K}$ in front of our custom Michelson interferometer, which is able to measure performance of the DM at actuator/segment level and at the entire mirror level, with a lateral resolution of $2 \mu \mathrm{m}$ and a sub-nanometer z-resolution. We tested the PTT 111 DM from Iris AO: an array of single crystalline silicon hexagonal mirrors with a pitch of $606 \mu \mathrm{m}$, able to move in tip, tilt, and piston (stroke $5-7 \mu \mathrm{m}$, tilt $\pm 5 \mathrm{mrad}$ ). The device could be operated successfully from ambient to $160 \mathrm{~K}$. An additional, mainly focus-like, $500 \mathrm{~nm}$ deformation of the entire mirror is measured at $160 \mathrm{~K}$; we were able to recover the best flat in cryo by correcting the focus and local tip-tilts on all segments, reaching $12 \mathrm{~nm}$ rms. Finally, the goal of these studies is to test DMs in cryo and vacuum conditions as well as to improve their architecture for stable operation in harsh environments.
\end{abstract}

Keywords: MEMS mirror arrays; MOEMS; cryogenic testing; adaptive optics; wavefront correction

\section{Introduction}

Wavefront correction is a key issue in a wide range of applications, from physics to biology or astronomy. Collimating and focusing with high accuracy a very large number of photons in high energy lasers, correcting the wavefront through diffuse or inhomogeneous media for sharp retinal and tissue imaging, or correcting, in closed loop, the atmospheric turbulence for revealing the faintest or most remote objects in the Universe, requires high performance wavefront correction systems.

In astronomy, high performance adaptive optical (AO) systems are being studied around the world for next generation instrumentation of $10 \mathrm{~m}$-class telescopes as well as for future extremely large optical telescopes. Adaptive optics systems are based on a combination of three elements: the wavefront sensor for measuring the shape of the wavefront arriving in the telescope; the deformable mirror for correcting the wavefront; and, finally, the real time computer for closing the loop of the system at a frequency ranging from 0.5 to $3 \mathrm{kHz}$, in order to follow the evolution of the atmospherical perturbations (Figure 1). 


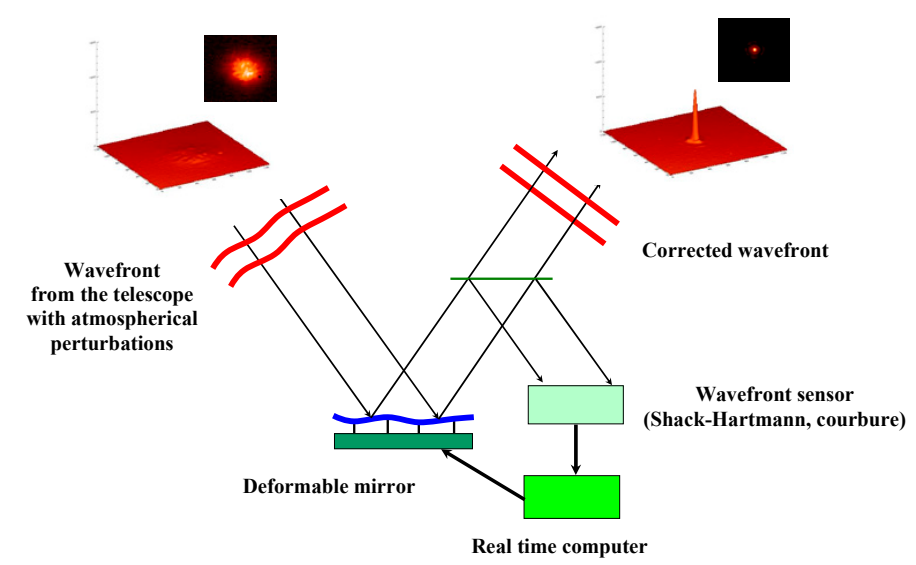

Figure 1. Schematic of a wavefront correction system.

Four main types of AO systems have been built or are under development: Single-Conjugate Adaptive Optics (SCAO), Multi-Conjugate Adaptive Optics (MCAO), Multi-Object Adaptive Optics (MOAO), and Extreme Adaptive Optics (ExAO). These AO systems are associated with different types of WaveFront Sensors (WFS), combined with natural guide stars or laser guide stars, and different architectures of Deformable Mirrors (DM). Numerous science cases will use these AO systems: SCAO, the "classical" AO system, will provide accurate narrow field imagery and spectroscopy; MCAO, wide field imagery and spectroscopy; $\mathrm{MOAO}$, distributed partial correction $\mathrm{AO}$, and high dynamic range $\mathrm{AO}$ for the detection and the study of circumstellar disks and extra-solar planets. Corrected fields will vary from few arcsec to several arcmin.

These systems require a large variety of deformable mirrors with very challenging parameters. For a $8 \mathrm{~m}$ telescope, the number of actuators varies from a few 10 up to 5000; these numbers increase impressively for a $40 \mathrm{~m}$ telescope, ranging from a few 100 to over 50,000 (the inter-actuator spacing from less than $200 \mu \mathrm{m}$ to $1 \mathrm{~mm}$, and the deformable mirror size from $10 \mathrm{~mm}$ to a few $100 \mathrm{~mm}$ ). Conventional technology cannot provide this wide range of deformable mirrors. The development of new technologies based on micro-opto-electro-mechanical systems (MOEMS) is promising for future deformable mirrors. The major advantages of the micro-deformable mirrors (MDM) are their compactness, scalability, and specific task customization using elementary building blocks. This technology permits the development of a complete generation of new mirrors. However this technology also has some limitations. For example, pupil diameter is an overall parameter and for a $40 \mathrm{~m}$ primary telescope, the internal pupil diameter cannot be reduced below $0.5 \mathrm{~m}$. According to the maximal size of the wafers (8 inches), a deformable mirror based on MOEMS technology cannot be built into one piece. New $\mathrm{AO}$ architectures have been proposed to avoid this limitation [1].

This new family of deformable mirrors will have also to fulfill some additional requirements: their ability to work in vacuum and at cryogenic temperatures. In order to study the early Universe, astronomers need to image and characterize astronomical objects (galaxies, quasars, large scale structures, etc.) in the infra-red part of the electromagnetic spectrum. At these wavelengths, the background noise of the instruments themselves must be reduced drastically by cooling them down at cryogenic temperatures, the longer the wavelength, the lower the temperature. Another challenge is to introduce MOEMS devices in future space instruments for reaching unprecedented performance. For all these applications, designing, testing, and operating MOEMS deformable mirrors in harsh environments (vacuums, cryogenic temperatures) is a critical issue.

For several years Laboratoire d'Astrophysique de Marseille (LAM) has been involved in the conception of new MOEMS devices as well as in the characterization of these components for the future instrumentation of ground-based and space telescopes. These studies include programmable slits for application in multi-object spectroscopy (JWST, European networks, EUCLID, BATMAN), deformable mirrors for adaptive optics, and programmable gratings for spectral tailoring. 
We are particularly engaged in a European development of micromirror arrays (MMA) called MIRA for generating reflective slit masks in future Multi-object spectroscopy (MOS) instruments; this technique is a powerful tool for space and ground-based telescopes for the study of the formation and evolution of galaxies. MMA with $100 \times 200 \mu \mathrm{m}^{2}$ single-crystal silicon micromirrors were successfully designed, fabricated, and tested. Arrays are composed of 2048 micromirrors $(32 \times 64)$ with a peak-to-valley deformation less than $10 \mathrm{~nm}$, and a tilt angle of $24^{\circ}$ for an actuation voltage of $130 \mathrm{~V}$. The micromirrors were actuated successfully before, during, and after cryogenic cooling, down to $162 \mathrm{~K}$. The micromirror surface deformation was measured at cryo and is below $30 \mathrm{~nm}$ peak-to-valley [2,3]. In order to fill large focal planes (mosaicing of several chips), we are currently developing large micromirror arrays integrated with their electronics.

LAM is also leading the conception and realization of new MOEMS-based instruments, including the development of the Digital-Micromirror-Device (DMD)-based MOS instrument, to be mounted on the Telescopio Nazionale Galileo (TNG) by mid-2018 and called BATMAN [4].

In this paper, we present the specific set-up for the interferometric characterization of a segmented deformable mirror from Iris $\mathrm{AO}$, in vacuum and at cryogenic temperatures. The results on the mirror surface operated from ambient down to $160 \mathrm{~K}$ are shown for the first time and analyzed.

\section{Deformable Mirrors}

Three main Micro-Deformable Mirrors (MDM) architectures are under study in different laboratories and companies. First, the bulk micro-machined continuous-membrane deformable mirror, studied by Delft University and OKO Company (Rijswijk, The Netherlands), is a combination of bulk silicon micromachining with standard electronics technology [5]. This mirror is formed by a thin flexible conducting membrane, coated with a reflective material, and stretched over an electrostatic electrode structure. This mirror shows very good mirror quality, but the mean deformed surface is a concave surface, and the number of actuators cannot be scalable to hundreds of electrodes. Second, the segmented, micro-electro-mechanical deformable mirror realized by Iris AO [6] consists of a set of segmented piston-tip-tilt moving surfaces, fabricated in dense array. Third, the surface micro-machined continuous-membrane deformable mirror made by Boston Micromachines Corporation (BMC) is based on a single compliant optical membrane supported by multiple attachments to an underlying array of surface-normal electrostatic actuators [7]. This device has been demonstrated recently in several AO systems, including the Gemini Planet Imager (GPI) instrument on Gemini telescope. The third concept shows limited strokes for large driving voltages, and the mirror surface quality may need further improvement for Extreme AO. All of these devices are based on silicon or polysilicon materials.

Two candidates, BMC deformable mirrors and Iris AO deformable mirrors, are foreseen for characterization in vacuum and at cryogenic temperatures, but none has yet been fully operated at cryogenic temperatures. Their architectures are presented in this paragraph.

\subsection{BMC Deformable Mirror}

BMC produces advanced MEMS deformable mirrors. The concept is based on an array of electrostatic actuators linked one by one to a continuous top mirror surface with etch holes (Figure 2). Their main parameters are approaching the requirements values, i.e., large number of actuators (up to 4096, see Figure 2), large stroke (up to $5.5 \mu \mathrm{m}$ for lower actuator count devices), and good surface quality.

BMC is currently conducting several actions for developing DMs devoted to space applications. In 2011, a classical DM has been tested with a Sounding Rocket in the US, in the Planetary Imaging Concept Testbed Using a Rocket Experiment (PICTURE). PICTURE-B has been launched in November 2015. This experiment measures directly optical light scattered by the debris disk around Epsilon-Eri star. In parallel, several studies have been engaged to modify the DM architecture and make it compatible with space environment [8]. 


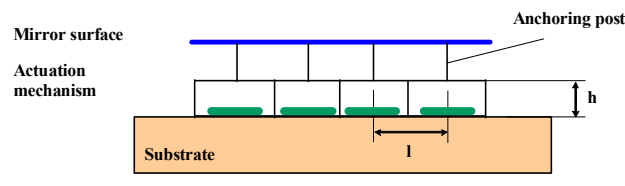

(a)

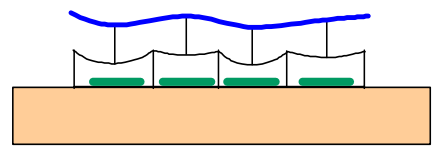

(b)



(c)

Figure 2. Continuous membrane Micro-Deformable Mirrors (MDM) from Boston Micromachines Corporation (BMC): schematic view of the (a) MDM non-actuated, (b) MDM actuated; (c) devices picture (courtesy from BMC).

\subsection{Iris AO Deformable Mirror}

Iris AO has been manufacturing piston-tilt-tilt (PTT) MEMS DMs with high-optical-quality mirror segments since 2002. University research that demonstrated the basic concept was first reported in 2001 [9] and early demonstrations of products were reported some years later [10]. Current products span apertures of $3.5 \mathrm{~mm}-7.7 \mathrm{~mm}$ with 111-489 actuators. A 939 actuator DM will be available in late 2017 and development is underway on a 3000 actuator PTT DM.

Figure 3 shows photographs of two different segmented DMs manufactured by Iris AO with 111 (PTT111) and 489 (PTT489) actuators. The DM segments are independent and are controlled by three actuators to give three degrees of freedom. Thus, the PTT111 DM has a total of 37 independent PTT segments and the PTT489 DM has 163. The PTT111 DM shown here is coated with a protected-silver coating. The PTT489 DM is shown with a gold coating. The coatings are typical coatings used for macroscale optics. They are much thicker than typical coatings used on MEMS and thin-membrane mirrors, so they are very robust. This is possible because the segment design and fabrication technology is very tolerant to various coatings. Even high-reflectance dielectric coatings for wavelengths ranging from $255 \mathrm{~nm}-1600 \mathrm{~nm}$ are available as options.

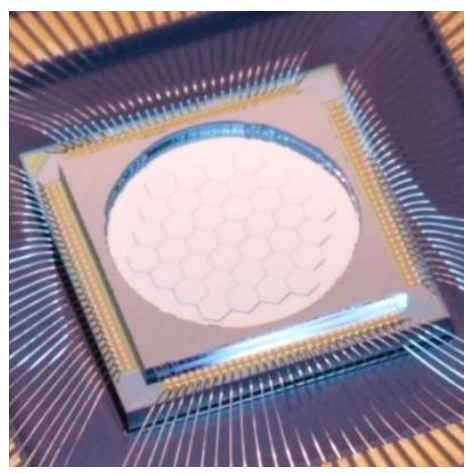

(a)

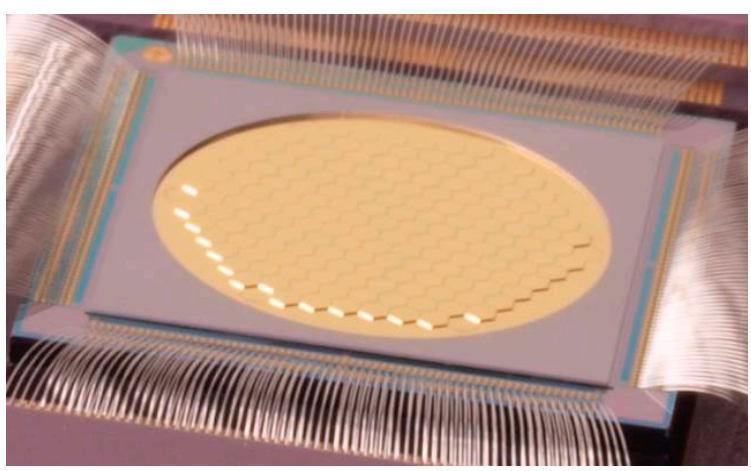

(b)

Figure 3. (a) Photograph of protected-silver coated PTT111 Deformable Mirror (DM) mounted in a ceramic package. The 111 actuator DM is comprised of 37 PTT segments tiled in a hex-packed array with an inscribed aperture of $3.5 \mathrm{~mm}$; (b) Photograph of gold-coated PTT489 DM. The 489 actuator DM is comprised of 163 PTT segments with an inscribed aperture of $7.7 \mathrm{~mm}$. The $700 \mu \mathrm{m}$ vertex-to-vertex segment footprint for the PTT111 and PTT489 DM are identical.

Figure 4 shows an exploded-view schematic diagram of one of the mirror segments. During manufacturing, the rigid mirror segments are bonded to actuator arrays at the chip level prior to 
the microstructure release step. After removing the sacrificial layer, the segments elevate above the underlying electrodes because of engineered residual stresses in the bimorph and actuator platform materials. The proprietary material stack is chosen to minimize elevation due to changes in temperature. The scaling in the vertical direction is highly exaggerated in Figure 4. In reality, the platform elevation is up to $40 \mu \mathrm{m}$ whereas the segments are $350 \mu \mathrm{m}$ on a side (700 $\mu \mathrm{m}$ across). The three underlying actuator electrodes enable piston-tip-tilt (PTT) positioning. Factory calibration and an open-loop controller conveniently convert from a desired position to actuator voltages [11].

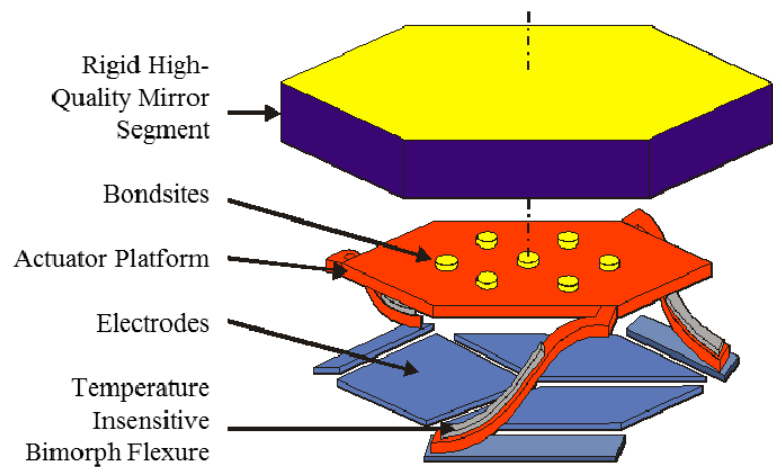

Figure 4. Exploded-view schematic diagram of a $700 \mu \mathrm{m}$ circumscribed diameter $(606.2 \mu \mathrm{m}$ flat-to-flat pitch) mirror segment. Scaling is highly exaggerated in the vertical direction. Actuator platform heights are in the range of $20-40 \mu \mathrm{m}$ for these segments, which enables $8 \mu \mathrm{m}$ of stroke.

The DM is manufactured using typical MEMS and integrated circuit materials such as polycrystalline silicon (polysilicon), silicon dioxides, silicon nitrides, and a proprietary bimorph material with similar coefficient of thermal expansion (CTE) to that of polysilicon. After the DM is fabricated using highly stable MEMS materials, it is mounted onto a ceramic pin-grid array (PGA) package using an epoxy. The DM is sealed in nitrogen by epoxying a cover window over the DM.

\section{Cryogenic Interferometric Test Set-Up}

Over the last few years, LAM has developed expertise in the characterization of micro-optical components, especially for small-scale deformation characterization on their surface from room temperature down to cryogenic temperatures. The aim is to be able to use these devices in all type of spectrographs, from ground-based to space telescopes as well as from Visible to IR instruments. The background of this test set-up development was in the framework of the NASA study of NIRSpec for the JWST.

\subsection{Interferometric Bench}

The measurement of the shape and the deformation parameters of the MOEMS devices is made thanks to an interferometric characterization bench, fully developed in-house (hardware and software). Characterizations are done in static or dynamic modes, including measurements of optical surface quality at different scales, actuator stroke, maximum mirror deformation, and cut-off frequency; the latest parameter has not yet been measured in this study.

This bench is a high-resolution and low-coherence Twyman-Green interferometer. It is a modular set-up around three main parts: illumination, interferometric cavity, and imaging system. The illumination is provided by a halogen lamp with an interference filter (typical example: $\lambda_{0}=650 \mathrm{~nm}$, $\Delta \lambda=10 \mathrm{~nm}$ ). Fixing the temporal light coherence eliminates all parasitic fringe patterns induced by classical high coherence sources such as lasers. The interferometric cavity is clear of any optical components in order to keep the highest optical quality by avoiding any differential aberration introduced by these additional elements. The imaging system offers two magnification configurations 
by a simple lens change: (1) a high in-plane resolution or (2) a large field of view authorizing either a very sharp (around $2 \mu \mathrm{m}$ ) analysis of the micro-mirror structure inside a small field (typically $1 \mathrm{~mm}$ ), or the whole device study with a larger size (up to $40 \mathrm{~mm}$ ).

Out-of-plane measurements are performed with phase-shifting interferometry showing very high resolution (standard deviation $<1 \mathrm{~nm}$ ). The bench is mounted on a damped optical table, and surrounded by a Plexiglas enclosure. Features such as optical quality or electro-mechanical behavior are extracted from these high precision three-dimensional component maps. Range is increased without loosing accuracy by using two-wavelength phase-shifting interferometry authorizing large steps measurements. Dynamic analysis like vibration mode and cut-off frequency could be also measured with time-averaged interferometry [12].

\subsection{Cryogenic Experiment}

In front of our interferometric setup has been installed a cryo chamber for reaching temperatures as low as $100 \mathrm{~K}$ when not loaded, using a cryogenic generator, and vacuum pressure in the range of $10^{-6}$ mbar (Figure 5a). The chamber is equipped with an internal screen radiatively insulating the sample from the chamber. Temperature sensors and local heaters are used for controlling the environment, and connected to a custom built control electronics and control-command software. The component is monitored continuously thanks to a glass window at the entrance of the chamber. As we are using low coherence interferometry, the fringe contrast can be kept at maximum by balancing the optical path in the reference arm of the interferometer with a compensation plate identical to the chamber window. In our set-up, the chamber window and the compensation plate have been manufactured at the same time in the same glass material [13].

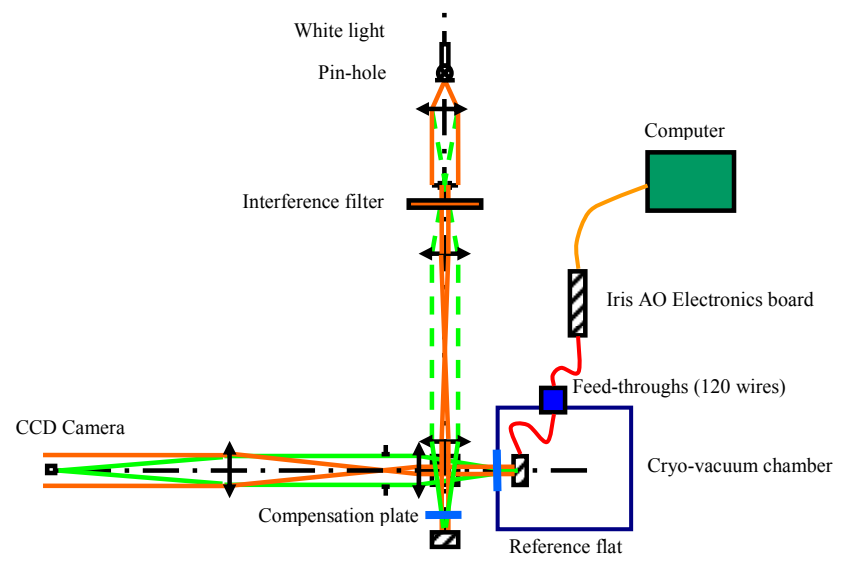

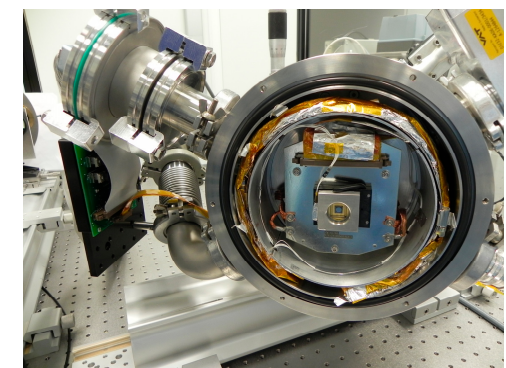

(b)

(a)

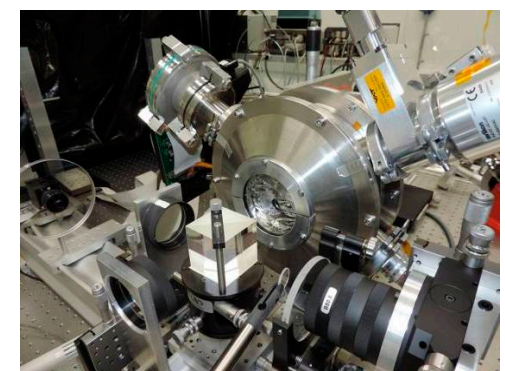

(c)

Figure 5. (a) Schematic description of our interferometric measurement set-up with the cryo-vacuum chamber; (b) PTT111 device mounted in the cryogenic chamber for characterization in a space environment; (c) Front window is closed and the cryogenic chamber is installed in front of our interferometric setup. The segmented deformable mirror could be successfully actuated before, during, and after cryogenic cooling at $160 \mathrm{~K}$. 


\subsection{PTT111 Mounting}

The PTT111 device is packaged in PGA chip carrier. The PGA is inserted in a ZIF-holder integrated on a PCB board. Large metallic surfaces on the PCB facilitate cooling down the system; eliminating the solder-stop layer eases outgassing of the PCB base material during evacuation of the chamber. The PCB itself is mounted via a fix-point-plane-plane attachment system to a solid aluminum block, the latter being interconnected to the cryo-generator (Figure $5 b$ ). Thick copper wires between the PCB and the aluminum block further enhance thermal transport between the sample chip and the cryostat. Two ribbon cables allow interconnecting up to 120 electrical wires through two 100-pin and 20-pin feed-throughs. Outside of the cryo chamber, the wires are connected to the Iris AO control electronics. Temperature sensors are connected to the aluminum block and to the window frame on top of the device.

The chamber is then closed by a flange and placed in front of the interferometer (Figure 5c). Along the reference path, two compensation plates are placed for compensating the chamber window (large plate) and the device window (small plate). By this way, we keep a high contrast for the interferometric fringes.

\section{PTT111 Surface Characterization}

These first experiments are done using an engineering grade device where segments \#23 and \#24 are inactive because of defects in the DM that occurred during manufacturing. The manufacturer calls these locked segments or lockouts as they are not controllable. Because the segments are independent, the defects only affect the locked segments and not neighboring one. The segment thickness is $25 \mu \mathrm{m}$ and the coating is protected silver. The maximum array stroke after flattening is $3.01 \mu \mathrm{m}$, and the maximum tilt angle is $5 \mathrm{mrad}$. Figure 6 is a picture of the device made on our bench (without the interferometric fringes). The two lockouts segments are at the upper right.



Figure 6. PTT111 device tested in our experiment (engineering grade device). Segments \#23 and \#24 (in the red circle) are lockouts.

The device is driven by the Graphical User Interface (GUI) provided by Iris AO for the integration and pre-characterization phases. The interferometric measurements are done with the LAM-developed software, in Matlab, and linked with the Matlab driver provided by Iris AO. The GUI is showing a view of the mirror with numbered segments, and the global Zernike coefficient as well as local (at segment level) piston/tip/tilt positions could be tuned for each segment.

A calibration step has been done by Iris AO for measuring each actuator response (3 actuators/segment). Then, a "best flat" condition is calculated in order to minimize the residual wavefront error on the surface, and applied. Figure 7 is a screen shot made at the beginning of the experiment when the best flat condition is applied; at the left hand side is the interferometric image 
of the mirror with the "best flat" condition; at the right bottom is the view of the Iris AO GUI for driving the device.

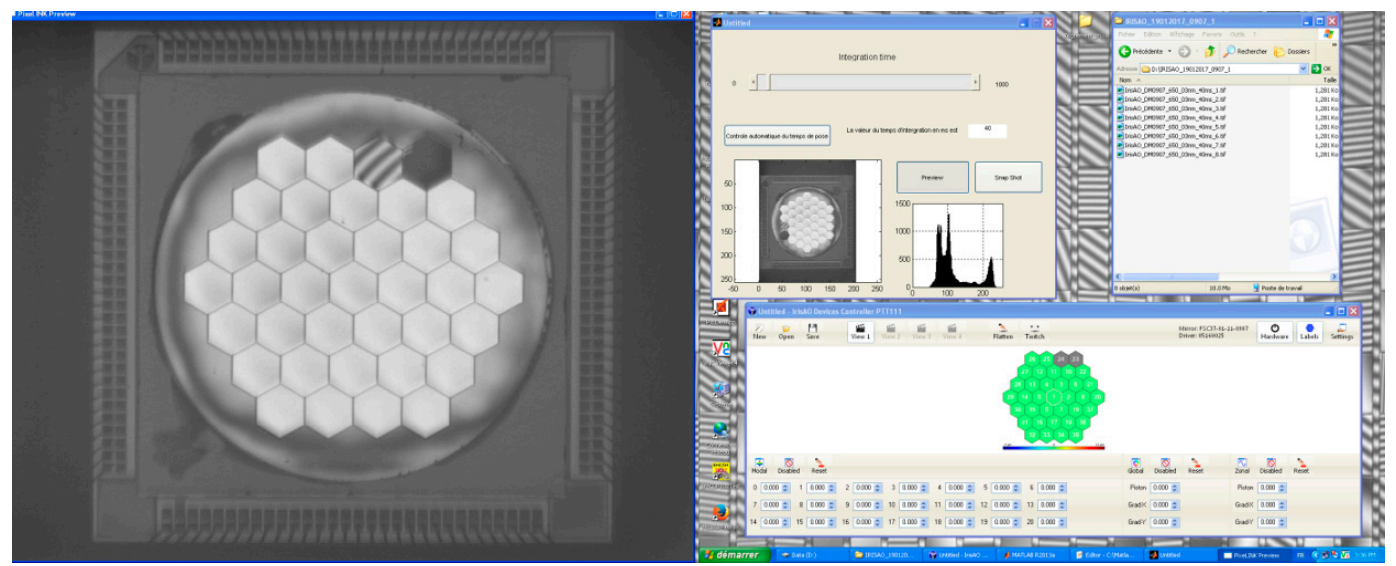

(a)

(b)

Figure 7. Screen shot during the experiment; (a) The interferometric image of the mirror when the "best flat" condition is applied; (b) The Iris AO GUI for driving the device.

We could then apply a series of commands to the mirror. In Figure 8, different mirror configurations are presented. From top left to bottom right, we can see:

a pure piston $(150 \mathrm{~nm})$ on the central M1 segment,

a global astigmatism on the mirror, using the global Zernike coefficient set at 0.125,

a series of three identical tilts on all segments in X direction, with $0.25 \mathrm{mrad}, 1.5 \mathrm{mrad}$, and $4.9 \mathrm{mrad}$ respectively.

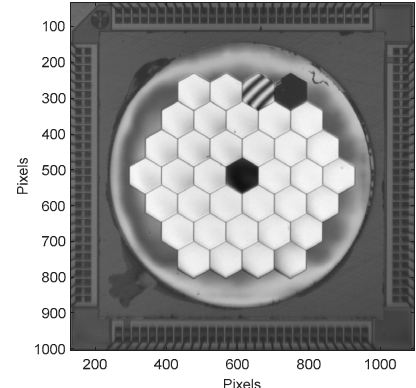

(a)

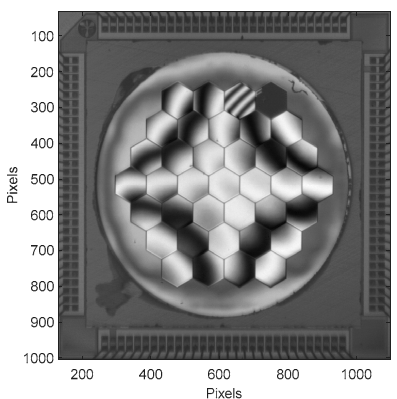

(b)

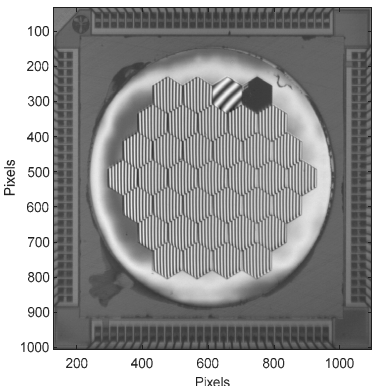

(e)

Figure 8. (a) $150 \mathrm{~nm}$ piston on segment\#1; (b) astigmatism (0.125 on Zernike coefficient); (c-e) Increasing tilt values along the $X$ direction are applied to all segments $(0.25,1.5$ and $4.9 \mathrm{mrad})$. 


\subsection{Best Flat at Ambient}

From the interferometric measurement, we obtain the surface deformation of the deformable mirror; in the whole paper, deformation values are always given in nanometers, and maps are displayed with a $\pm 250 \mathrm{~nm}$ range for better comparison between related Figures 9, 11, and 13a-15a.

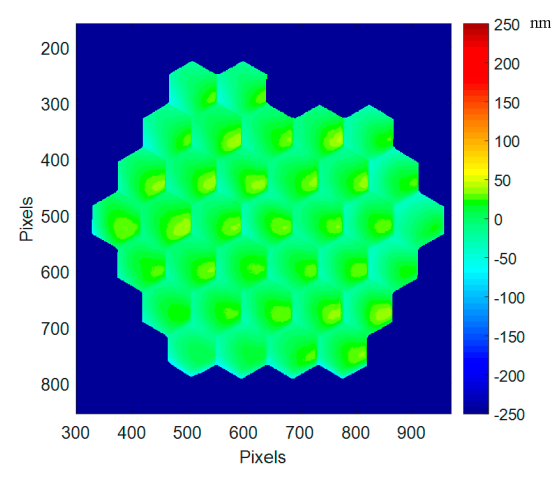

Figure 9. Best flat surface deformation at ambient (17 nm RMS, $123 \mathrm{~nm} \mathrm{PtV)}$ when the best flat condition calibrated by Iris AO is applied; the gravity effect has not been removed.

The best flat residual over the whole mirror is very good with $17 \mathrm{~nm}$ RMS, $123 \mathrm{~nm} \mathrm{PtV}$ (Figure 9). Note that this best flat provided by Iris AO is not corrected from the gravity effect occurring in our experiment, as the PTT111 is mounted in vertical position.

This result shows the high quality of the mirror architecture and of the fabrication process. This flatness is a combination of a very good reproducibility of the actuator platform position after his elevation thanks to the bimorph flexures (Figure 4), and the choice of thick single-crystalline Silicon for the segment material. This position is very stable; long term measurement has been done at ambient on position stability [6] and reproducibility, but this has not been done yet in cryo.

At segment level, the residual wavefront error is with a slight convex shape, observed on most of the segments (see Section 4.6).

We measure the mirror surface shape with high accuracy and sort out tilt and piston values for wach segment. They are displayed on segment maps in Figure 10. Over the maps, the tilts have a value of $32 \mu \mathrm{rad}$ RMS $(140 \mu \mathrm{rad} \mathrm{PtV})$ while the pistons expands on $6.2 \mathrm{~nm}$ RMS (28 nm PtV). The residual tilts are mainly due to the gravity effect as the device is mounted in vertical position in our measurement set-up.

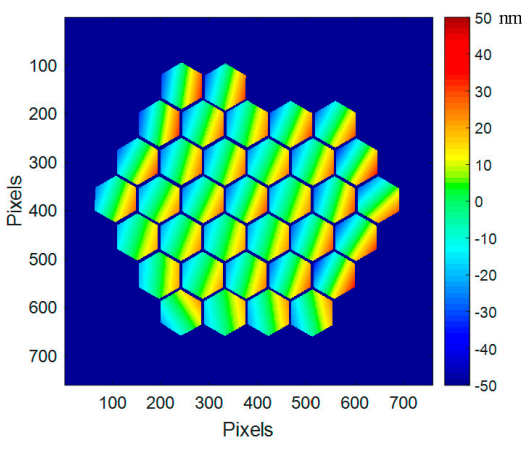

(a)

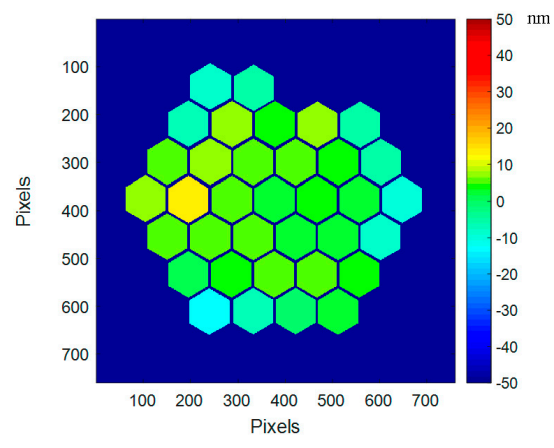

(b)

Figure 10. Residual tip-tilts (a) and pistons (b) at ambient, when the best flat condition from Iris AO is applied; the gravity effect has not been removed. 


\subsection{Best Flat at $160 \mathrm{~K}$, First Run}

The device is then cooled down slowly from ambient temperature (293 K) down to $160 \mathrm{~K}$, with the device constantly operating in its best flat condition. The PTT111 device is operating properly at all temperatures between $293 \mathrm{~K}$ and $160 \mathrm{~K}$, and in vacuum.

Every $10 \mathrm{~K}$ an interferometric measurement is done in order to follow the differential deformation of the mirror at whole mirror level as well as at segment level. Several patterns are applied and measured in order to see the ability of the device to behave as at room temperature; the applied "patterns" are best flat, pure pistons on some segments, and different tilts on the segments. Due to the vibrations induced by the cryo pump on the sample, we have to stop it during the measurement, leading to a limited increase of the temperature during the measurement duration. Phase shifting interferometry parameters have been adjusted in order to minimize the measurement time to a few minutes.

In Figure 11, the best flat surface deformation at cryo $(160 \mathrm{~K})$ is given with the original best flat condition as calibrated at ambient. A global convex deformation is observed reaching a deformation of $86 \mathrm{~nm}$ RMS, $501 \mathrm{~nm}$ PtV. Some additional deformations (mainly tilts) are observed on some segments at the upper left side (segments \#26, 27, and 28).

The global convex shape in cryo is due to the packaging "shrinking" in cryo. The Coefficient of Thermal Expansion (CTE) mismatch between die/package materials induces a global effect on the mirror when cooled down at $160 \mathrm{~K}$.

The mirror is operating perfectly in cryo, and a "new" best flat condition will be developed and described in the next paragraphs.

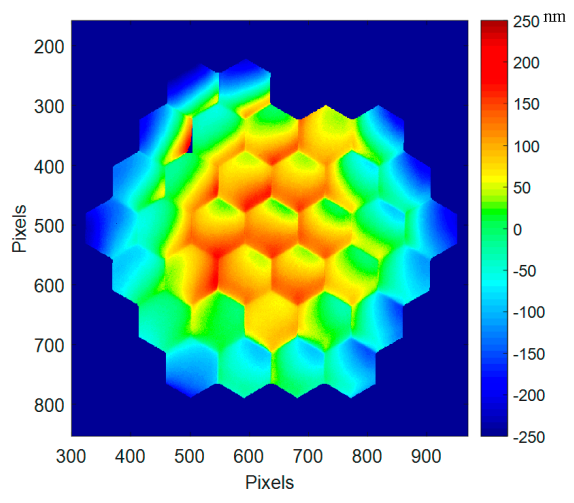

Figure 11. Best flat mirror deformation at cryo (160 K), first run, with the original best flat calibrated at ambient (86 nm RMS, $501 \mathrm{~nm}$ PtV).

We measure and sort out for each segment tilt and piston values. They are displayed on segment maps and shown in graphs, in Figure 12. The tilts have a value of $200 \mu \mathrm{rad}$ RMS (950 $\mu \mathrm{rad} \mathrm{PtV})$ while the pistons expands on $74 \mathrm{~nm}$ RMS (239 nm PtV). Tilts and pistons are not behaving the same way. Pistons are behaving within the three concentric rings of PTT111 37 segments, with a common motion for the central area (segments \#1 to 7), the middle ring (segments \#8 to 19), and the outer ring (segments \#20 to 37); in Figure 12, residual pistons at ambient (black signs) are $6.2 \mathrm{~nm} \mathrm{RMS} \mathrm{(28} \mathrm{nm} \mathrm{PtV),} \mathrm{and}$ $74 \mathrm{~nm}$ RMS (239 nm PTV) in cryo (red signs); this effect is clearly related to the shrinkage of the overall device. As for the tilts, they don't show a clear pattern; they are scattered away from the original positions at ambient. In Figure 12, residual tilts at ambient (black signs) are $32 \mu \mathrm{rad}$ RMS (140 $\mu \mathrm{rad}$ $\mathrm{PtV}$ ), and $200 \mu \mathrm{rad}$ RMS (950 $\mu \mathrm{rad}$ PTV) in cryo (red signs); this differential evolution is possibly due to the different modification of the complex structure underneath each segment: the actuator platform, the bonding pads, the mirror segment, the coating, and the underlying 3 legs bimorph structure. 

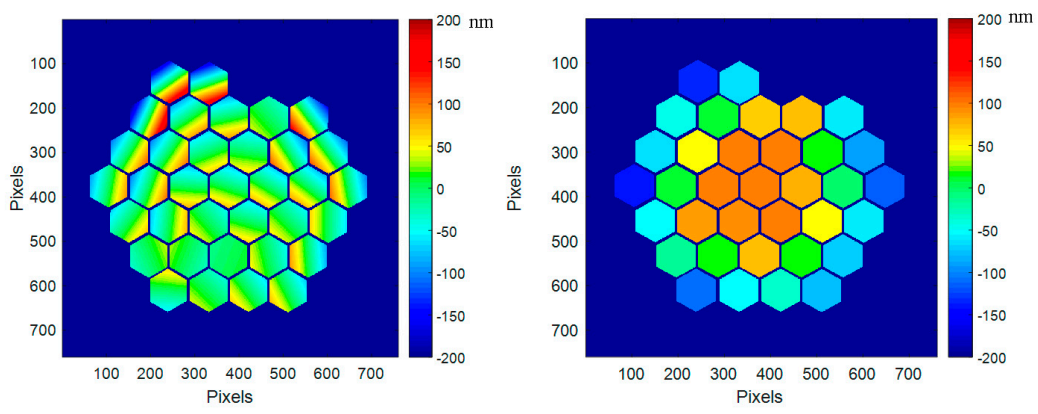

(a)
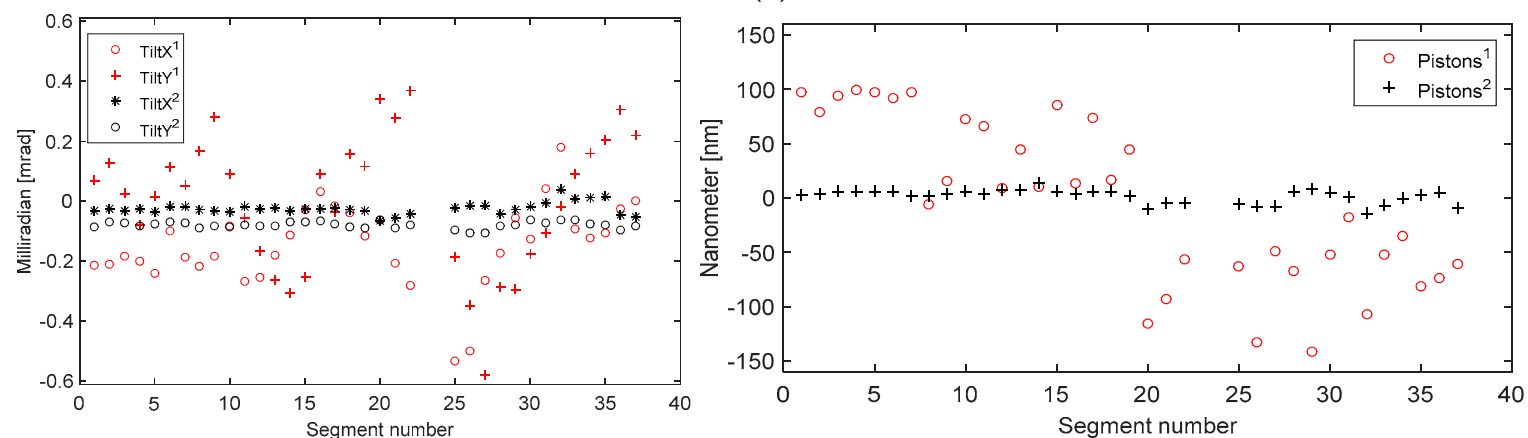

(b)

Figure 12. (a) Segment maps of the residual tip-tilts and pistons at cryo (160 K), first run; (b) Values for each of the 37 segments of PTT111 (in red tilt X and tilt $Y$ at cryo, in black, for reference, values at ambient). Residual tilts at ambient (black signs) are $32 \mu \mathrm{rad}$ RMS (140 $\mu \mathrm{rad}$ PtV), and $200 \mu \mathrm{rad}$ RMS (950 $\mu$ rad PTV) in cryo (red signs). Residual pistons at ambient (black signs) are $6.2 \mathrm{~nm}$ RMS (28 nm $\mathrm{PtV}$ ), and $74 \mathrm{~nm}$ RMS (239 nm PTV) in cryo (red signs).

These effects will be corrected in a second step (see Section 4.4).

\subsection{Best Flat at Ambient (Back 1)}

When the device is warmed up back to ambient temperature we measure its surface shape in the original best flat mode again, and we get a slightly different result, as revealed in Figure 13.

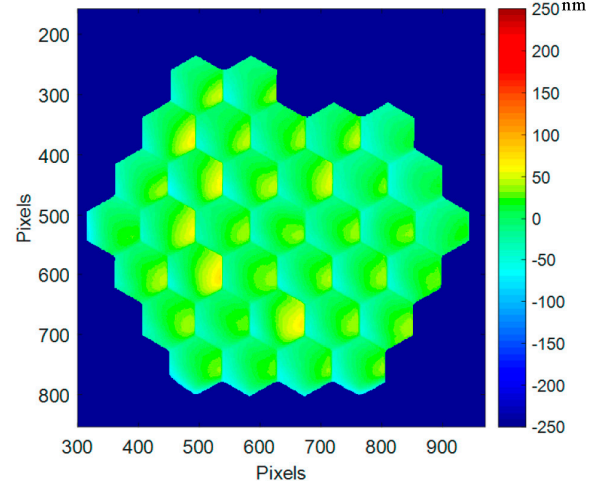

(a)

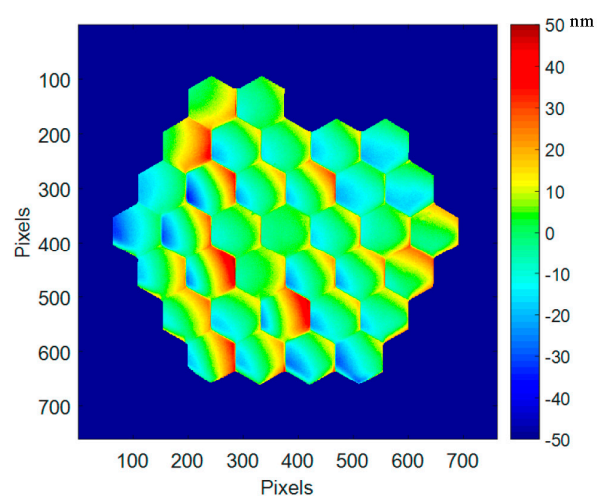

(b)

Figure 13. Mirror surface deformation at ambient ( $293 \mathrm{~K})$ when best flat Iris AO condition is applied, after one cooling cycle: (a) Surface deformation map (22 nm RMS, $123 \mathrm{~nm} \mathrm{PtV);} \mathrm{(b)} \mathrm{Difference} \mathrm{between}$ the two surface deformation maps before and after one cooling cycle. 
The degradation of the surface deformation is going up from $17 \mathrm{~nm}$ to $22 \mathrm{~nm}$ RMS (123 nm to $152 \mathrm{~nm} \mathrm{PtV})$, the differences being mainly due to the additional tilts on each segment by $0.15 \mathrm{mrad}$ $\mathrm{PtV}$ over the device, while the pistons stay within the same range as before the cooling cycle. This is probably due to the stress relaxation process in the complex structure supporting each segment, leading to a slightly different evolution from segment to segment. As for the segment mirror surface, there is no difference (below $1 \mathrm{~nm}$ ), thanks to the high quality of the segment made of single-crystalline silicon material. This effect could be described as an annealing process.

In order to improve the best flat quality, we decided to develop an improved best flat procedure by measuring, with high accuracy, the tip-tilt and piston residuals and combining them with the original best flat values calibrated by Iris AO. We then obtain the improved best flat shown in Figure 14 . The mirror surface deformation is then as low as $10 \mathrm{~nm}$ RMS, $79 \mathrm{~nm} \mathrm{PtV}$.

In the graphs of Figure 14, residual tilts with improved best flat (red signs) are $6.5 \mu \mathrm{rad}$ RMS (36 $\mu \mathrm{rad}$ PtV), while they were $32 \mu \mathrm{rad}$ RMS (140 $\mu \mathrm{rad}$ PTV) with the original best flat (black signs). Residual pistons with improved best flat (red signs) are $1.6 \mathrm{~nm}$ RMS $(7 \mathrm{~nm} \mathrm{PtV})$, while they were $6.2 \mathrm{~nm}$ RMS ( $28 \mathrm{~nm}$ PtV) with the original best flat (black signs).

Improved best flat condition will be used in the following experiments.

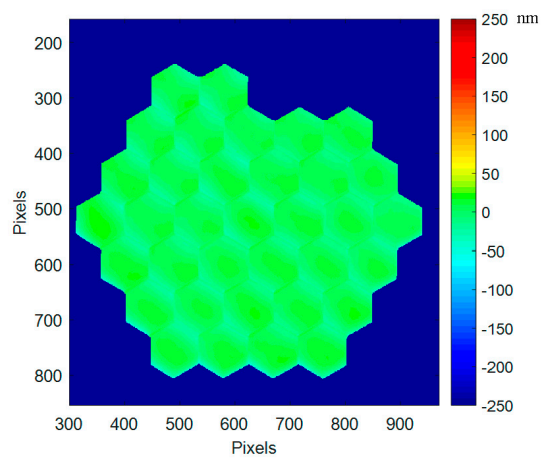

(a)
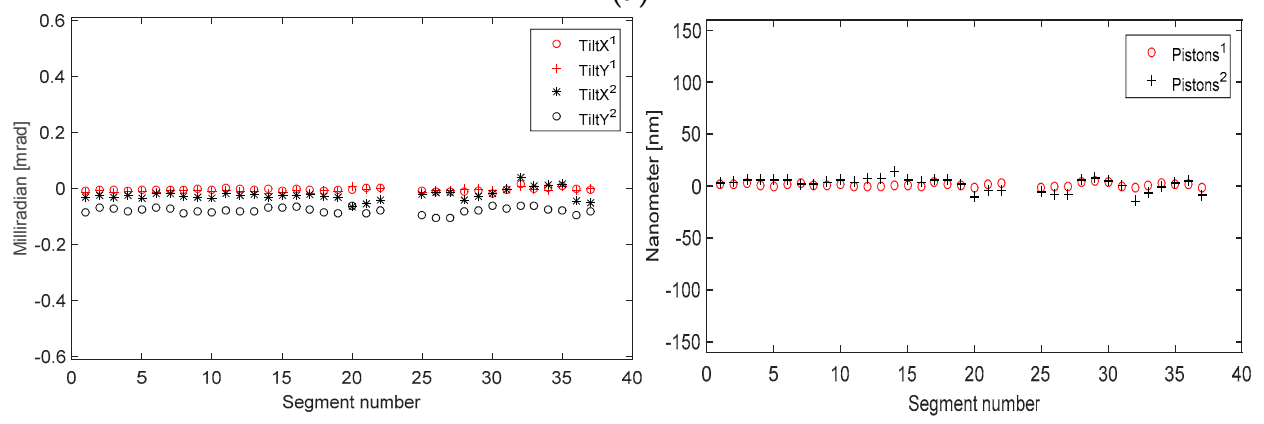

(b)

Figure 14. Mirror surface deformation at ambient (293 K) when improved best flat condition is applied, after one cycle of cooling: (a) Surface deformation map (10 nm RMS, $79 \mathrm{~nm} \mathrm{PtV);} \mathrm{(b)} \mathrm{Residual} \mathrm{tilts}$ and pistons values for each of the 37 segments of PTT111. Residual tilts with improved best flat (red signs) are $6.5 \mu \mathrm{rad}$ RMS (36 $\mu \mathrm{rad} \mathrm{PtV})$, while they were $32 \mu \mathrm{rad}$ RMS (140 $\mu \mathrm{rad}$ PTV) with the original best flat (black signs). Residual pistons with improved best flat (red signs) are $1.6 \mathrm{~nm} \mathrm{RMS} \mathrm{(7} \mathrm{nm} \mathrm{PtV),}$ while they were $6.2 \mathrm{~nm}$ RMS (28 nm PtV) with the original best flat (black signs).

\subsection{Best Flat at 160 K, Second Run}

In order to demonstrate full operation of PTT111 at cryogenic temperature, we decide to cool down the device and optimize in-situ all actuators for generating a cryo best flat.

Our strategy is a weighted addition of the consecutive measurement residual errors and, using Iris AO electronics, we are loading these calculated values actuator by actuator, departing from 
the original values provided by Iris $\mathrm{AO}$, and applyling them to the device. Our cryo best flat condition is a combination of [best flat calibrated by Iris-AO at ambient, improved best flat at ambient, improved best flat in cryo (first run), improved best flat in cryo (second run)]. Then, in a single measurement step and applying this best flat condition, we got, at $160 \mathrm{~K}$, a mirror surface deformation as low as 12 nm RMS, 113 nm PtV (Figure 15).

Our cryo best flat at $160 \mathrm{~K}$ is then very close to our improved best flat at ambient $(293 \mathrm{~K})$, showing our ability to operate properly PTT111 device in cryo. The deformation difference is $2 \mathrm{~nm}$ RMS, $34 \mathrm{~nm}$ $\mathrm{PtV}$ between $160 \mathrm{~K}$ and $293 \mathrm{~K}$. This additional deformation is due mainly to the mirror segment deformation, as revealed in the following Section 4.6.



(a)

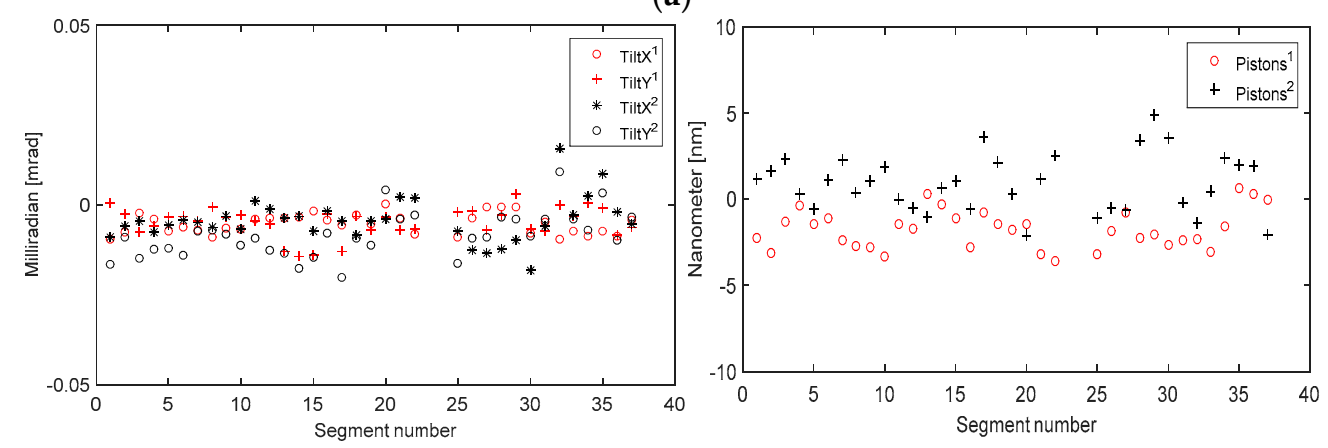

(b)

Figure 15. Mirror surface deformation at cryo $(160 \mathrm{~K})$, second run, when cryo best flat condition is applied: (a) Surface deformation map (12 nm RMS, $113 \mathrm{~nm} \mathrm{PtV);} \mathrm{(b)} \mathrm{Residual} \mathrm{tilts} \mathrm{and} \mathrm{pistons} \mathrm{values}$ for each of the 37 segments of PTT111. Residual tilts with cryo best flat (red signs) are $3.5 \mu$ rad RMS $(17 \mu \mathrm{rad} \mathrm{PtV})$, while they were $6.5 \mu \mathrm{rad}$ RMS $(36 \mu \mathrm{rad} \mathrm{PtV})$ with the improved best flat at ambient (black signs). Residual pistons with cryo best flat (red signs) are $1.2 \mathrm{~nm} \mathrm{rms}(4.3 \mathrm{~nm} \mathrm{PtV})$, while they were $1.6 \mathrm{~nm}$ RMS (7 nm PtV) with the improved best flat at ambient (black signs).

In the graphs of Figure 15, residual tilts with cryo best flat (red signs) are $3.5 \mu$ rad RMS (17 $\mu$ rad $\mathrm{PtV})$, while they were $6.5 \mu \mathrm{rad}$ RMS (36 $\mu \mathrm{rad} \mathrm{PtV})$ with the improved best flat at ambient (black signs). Residual pistons with cryo best flat (red signs) are $1.2 \mathrm{~nm} \mathrm{rms} \mathrm{(4.3} \mathrm{nm} \mathrm{PtV),} \mathrm{while} \mathrm{they} \mathrm{were} 1.6 \mathrm{~nm}$ RMS (7 nm PtV) with the improved best flat at ambient (black signs).

A second loop of best flat optimisation is useless as the remaining mirror surface deformation is only due to the contributions of individual segment deformations; this is clearly visible in the surface deformation map of Figure 15.

The additional deformation of PTT111 at cryo is $501 \mathrm{~nm} \mathrm{PtV} \mathrm{(Figure} \mathrm{11).} \mathrm{The} \mathrm{cryo} \mathrm{best} \mathrm{flat}$ is compensating this deformation, minimizing the whole mirror deformation, down to $12 \mathrm{~nm}$ RMS $(123 \mathrm{~nm} \mathrm{PtV})$. The maximum stroke of this device being $3.01 \mu \mathrm{m}$ at ambient, then the operational stroke is reduced to $2.5 \mu \mathrm{m}$ at cryo (16.7\% stroke reduction). 


\subsection{Best Flat at Ambient (Back 2)}

When the device is warmed up back to ambient temperature we measure its surface shape in the improved best flat mode again, and we get nearly exactly the same surface, as shown in Figure 14. The device seems to now be stabilized with no evolution after a complete cycle at cryogenic temperature.

A thermal cycling test might be useful to confirm this result.

\subsection{Analysis at Segment Level}

Thanks to our set-up spatial resolution, we have several thousand measurement points per segment. It is then possible to measure, at segment level, the deformation induced by the strong temperature change from ambient to cryo. Figure 16a,b are identical to Figures $14 \mathrm{a}$ and $15 \mathrm{a}$, with the mirror segment marked, and an expended scale for revealing the local effects on each segment. The surface deformation by segments at ambient (values 2 in blue) has a mean value of $7.2 \mathrm{~nm}$ with a standard deviation of $1.5 \mathrm{~nm}$, and at $160 \mathrm{~K}$ (values 1 in red), a mean value of $8.5 \mathrm{~nm}$ with a standard deviation of $1.6 \mathrm{~nm}$.

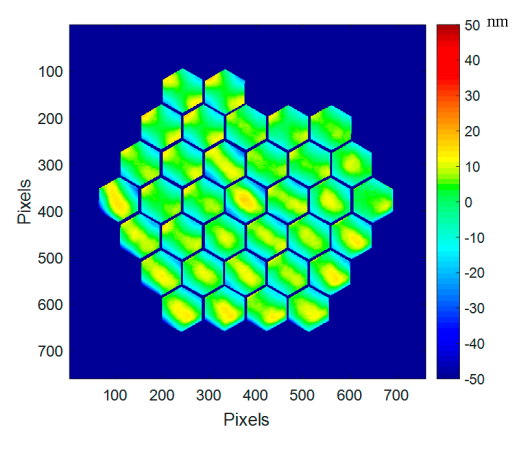

(a)

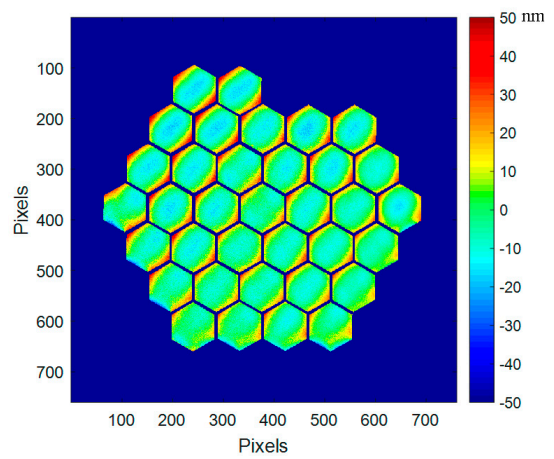

(b)

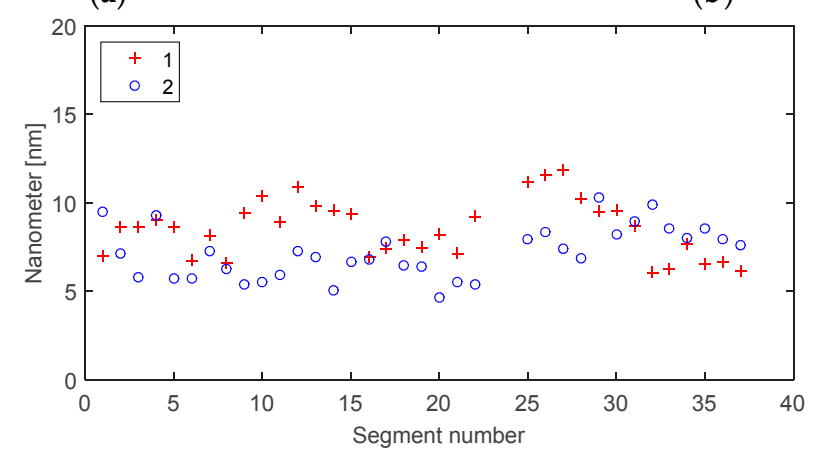

(c)

Figure 16. DM segments surface deformation: (a) At ambient (293 K), $5 \mathrm{~nm}$ RMS, $24 \mathrm{~nm} \mathrm{PtV;} \mathrm{(b)} \mathrm{At} 160 \mathrm{~K}$ $8 \mathrm{~nm}$ RMS, $47 \mathrm{~nm} \mathrm{PtV}$; (c) Surface deformation by segments at ambient (values 2 in blue) has a mean value of $7.2 \mathrm{~nm}$ with a standard deviation of $1.5 \mathrm{~nm}$, and at $160 \mathrm{~K}$ (values 1 in red), a mean value of $8.5 \mathrm{~nm}$ with a standard deviation of $1.6 \mathrm{~nm}$.

By selecting a typical segment, a closer analysis of the segment evolution at cryogenic temperature could be done, especially on its shape. In Figure 17, on segment \#21, we can clearly see that the convex cylindrical shape at ambient is changing to an astigmatic concave shape at cryo. At ambient (293 K), the segment surface deformation is $5 \mathrm{~nm}$ RMS (24 nm PtV), while at $160 \mathrm{~K}$ the deformation is still low, at $8 \mathrm{~nm}$ RMS (47 $\mathrm{nm} \mathrm{PtV).}$

A very interesting feature is observed when looking at the deformation difference between ambient and $160 \mathrm{~K}$ (Figure 17c): it reveals a pure concave axisymetrical change of $4.9 \mathrm{~nm}$ RMS (71 nm PtV). This is due to the CTE mismatch between the single-crystalline silicon and the 
silver-protected coating deposited on top of the segment. All segments are behaving in the same way as shown in Figure 18.

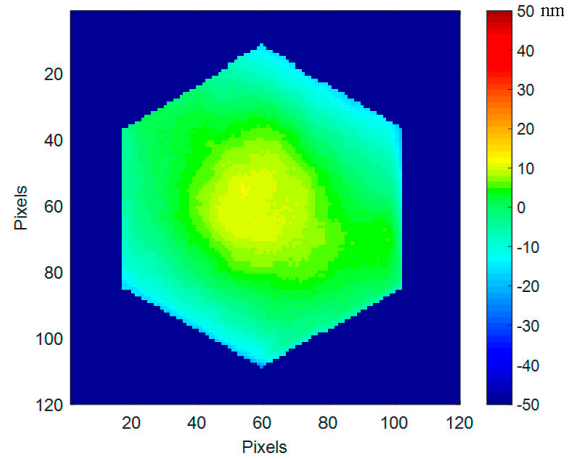

(a)

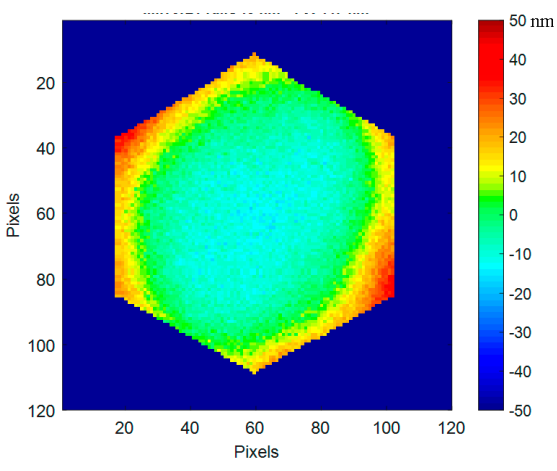

(b)

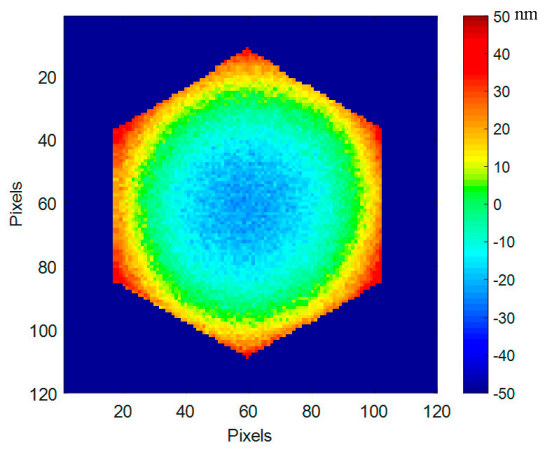

(c)

Figure 17. DM segment \#21 surface deformation: (a) At ambient (293 K), $5 \mathrm{~nm} \mathrm{RMS,} 24 \mathrm{~nm} \mathrm{PtV;}$ (b) At $160 \mathrm{~K} 8 \mathrm{~nm}$ RMS, $47 \mathrm{~nm} \mathrm{PtV;} \mathrm{(c)} \mathrm{Deformation} \mathrm{difference} \mathrm{between} \mathrm{ambient} \mathrm{and} 160 \mathrm{~K}, 4.9 \mathrm{~nm}$ RMS, $71 \mathrm{~nm} \mathrm{PtV.}$

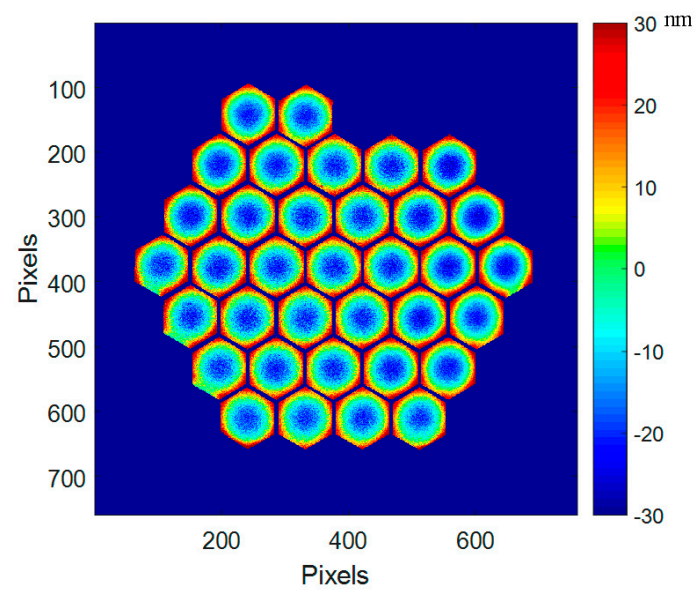

Figure 18. Segment deformation induced by the temperature change between ambient and cryo (160 K); map of all segments of PTT111.

The mean deformation at ambient is in the range of $25 \mathrm{~nm}$, while it rises to $50 \mathrm{~nm}$ in cryo. This deformation difference is still within the requirement of almost all foreseen wavefront correction systems. This deformation difference at segment level is the major contribution to the whole mirror surface deformation, as described in Section 4.4. 


\section{Conclusions}

Innovative wavefront correction systems in existing telescopes on the ground and in space, as well as in the future ELTs, need efficient MOEMS Deformable Mirrors (DM) able to perform at room temperature as well as in cryogenic and vacuum environments.

Using a specific interferometric bench coupled with a cryo-vacuum chamber, a PTT 111 DM from Iris AO has been successfully tested from ambient temperature to $160 \mathrm{~K}$. The device is properly operating in cryo, revealing an additional, mainly focus-like, $500 \mathrm{~nm}$ deformation at $160 \mathrm{~K}$; we were able to recover the best flat in cryo by correcting the focus and local tip-tilts on all segments, reaching $12 \mathrm{~nm}$ RMS on the entire surface.

Tests on DMs with different mirror thicknesses $(25 \mu \mathrm{m}$ and $50 \mu \mathrm{m})$ and different coatings (silver and gold) are currently under way.

Finally, the goal of these studies is to test DMs in cryo and vacuum conditions as well as to improve their architecture for stable operation in a harsh environment.

Author Contributions: F.Z. and M.H. supervised the work; M.H., F.M. and A.T. provided the devices and their driving electronics; P.L., F.Z. and R.B. designed and realized the experimental set-up; P.L. and F.Z. performed the experiments; F.Z. and P.L. carried out calculations and data treatment for the results interpretation; F.Z., P.L. and M.H. analyzed the results; F.Z. wrote the paper, and M.H. and P.L. contributed for some paragraphs.

Conflicts of Interest: The authors declare no conflict of interest.

\section{References}

1. Zamkotsian, F.; Dohlen, K. Prospects for MOEMS-based adaptive optical systems on extremely large telescopes. In Proceedings of the Conference Beyond Conventional Adaptive Optics, Venice, Italy, 7-10 May 2001.

2. Waldis, S.; Zamkotsian, F.; Clerc, P.-A.; Noell, W.; Zickar, M.; De Rooij, N. Arrays of high tilt-angle micromirrors for multiobject spectroscopy. IEEE J. Sel. Top. Quantum Electron. 2007, 13, 168-176. [CrossRef]

3. Canonica, M.; Zamkotsian, F.; Lanzoni, P.; Noell, W.; de Rooij, N. The two-dimensional array of 2048 tilting micromirrors for astronomical spectroscopy. J. Micromech. Microeng. 2013, 23, 055009. [CrossRef]

4. Zamkotsian, F.; Ramarijaona, H.; Moschetti, M.; Lanzoni, P.; Riva, M.; Tchoubaklian, N.; Jaquet, M.; Spano, P.; Bon, W.; Alata, R.; et al. Building BATMAN: A new generation spectro-imager on TNG telescope. In Proceedings of the SPIE Conference on Astronomical Instrumentation 2016 (Proc. SPIE 9908), Edinburgh, UK, 26 June-1 July 2016.

5. Vdovin, G.; Middelhoek, S.; Sarro, P.M. Technology and applications of micromachined silicon adaptive mirrors. Opt. Eng. 1997, 36, 1382-1390. [CrossRef]

6. Helmbrecht, M.A.; He, M.; Kempf, C.J.; Marchis, F. Long-term stability and temperature variability of Iris AO segmented MEMS deformable mirrors. In Proceedings of the SPIE 9909 Conference on Adaptive Optics Systems V, Edinburgh, UK, 26 June 2016.

7. Cornelissen, S.; Bifano, T.G. Advances in MEMS deformable mirror development for astronomical adaptive optics. In Proceedings of the SPIE 8253 Conference on MOEMS, San Francisco, CA, USA, 21-26 January 2012.

8. Bierden, P. MEMS deformable mirrors for space imaging. In Proceedings of the ESA Workshop on Innovative Technologies for Space Optics, Noordwijk, The Netherlands, 23-26 November 2015.

9. Helmbrecht, M.A.; Srinivasan, U.; Rembe, C.; Howe, R.T.; Muller, R.S. Micromirrors for adaptive-optics arrays, transducers 2001. In Proceedings of the 11th International Conference on Solid State Sensors and Actuators, Munich, Germany, 10-14 June 2001; pp. 1290-1293.

10. Helmbrecht, M.A.; Juneau, T.; Hart, M.; Doble, N. Performance of a high-stroke, segmented MEMS deformable-mirror technology. In Proceedings of the SPIE 6113 Conference on MEMS/MOEMS Components and Their Applications III, San Jose, CA, USA, 21 January 2006.

11. Helmbrecht, M.A.; Juneau, T. Piston-tip-tilt positioning of a segmented MEMS deformable mirror. In Proceedings of the SPIE 6467 Conference on MEMS Adaptive Optics, San Jose, CA, USA, 9 February 2007. 
12. Liotard, A.; Zamkotsian, F. Static and dynamic micro-deformable mirror characterization by phase-shifting and time-averaged interferometry. In Proceedings of the SPIE 5494 Conference on Astronomical Telescopes and Instrumentation, Glasgow, UK, 21-25 June 2004.

13. Zamkotsian, F.; Grassi, E.; Waldis, S.; Barette, R.; Lanzoni, P.; Fabron, C.; Noell, W.; de Rooij, N. Interferometric characterization of MOEMS devices in cryogenic environment for astronomical instrumentation. In Proceedings of the SPIE 6884 Conference on Reliability, Packaging, Testing, and Characterization of MEMS/MOEMS VII, San Jose, CA, USA, 7 February 2008.

(c) 2017 by the authors. Licensee MDPI, Basel, Switzerland. This article is an open access article distributed under the terms and conditions of the Creative Commons Attribution (CC BY) license (http://creativecommons.org/licenses/by/4.0/). 